\title{
Defect formation during preforming of a bi-axial non-crimp fabric with a pillar stitch pattern
}

S Chen, O P L McGregor, L T Harper*, A Endruweit, N A Warrior

Composites Research Group, Faculty of Engineering,

University of Nottingham, UK, NG7 2RD

*lee.harper@nottingham.ac.uk, Tel +44 (0)115 9513823

\begin{abstract}
To capture the asymmetrical shear behaviour of a bi-axial NCF with a pillar stitch, a nonorthogonal constitutive model was developed and implemented in finite element forming simulations. Preforming experiments indicate that the local distribution of defects is significantly different on both sides of each bi-axial ply, with two different defect mechanisms observed. Correlation with simulation results indicates that one defect type is caused by excessive shear, inducing out-of-plane wrinkling in regions of positive shear (macro-scale wrinkling). The other defect type is caused by fibre compression, inducing inplane wrinkling in regions of negative shear (meso-scale wrinkling). Local distributions of shear angle and wrinkling strain were used to determine the wrinkling mode and to confirm the corresponding defect mechanism. Results indicate that simulations based on the advanced constitutive model can predict local shear angles within $\pm 5^{\circ}$ of experimental values and that predicted wrinkling positions and defect types correlate well with the experiments.
\end{abstract}

\section{Keywords}

A. Fabrics/textiles; B. Defect; C. Finite element analysis (FEA); E. Forming; E. Preform 


\section{Introduction}

Non-Crimp Fabrics (NCF) offer increased stiffness and strength over frequently used woven fibre architectures [1] as reinforcement in composite structures, but have been shown to be more difficult to form into 3D shapes [2]. Bi-axial NCFs are composed of two layers of aligned yarns, typically at $0^{\circ} / 90^{\circ}$ or $\pm 45^{\circ}$, which are bonded together by through-thickness stitches. The stitch pattern can be adapted to either prevent unwanted shear deformation during handling through improved stability, or improve drapeability through relaxation of inplane constraints. In addition, multiple plies can be bonded together using local supplementary stitches to aid automated forming of complex lay-ups [3, 4].

The dominant forming mechanism of bi-axial NCFs is in-plane shear deformation, similar to other bi-directional materials such as woven fabrics. However, the mechanisms of shear are different, as the mesoscopic architecture of an NCF is established by introducing intra-ply stitches rather than by interlacing the primary yarns. In addition to rotation of the primary yarns, tension in the in-plane segments of the intra-ply stitches contributes to the shear resistance of the NCF. Inter-yarn friction also provides some resistance to shear deformation during yarn rotation. The orientation of the in-plane segments of the stitches relative to the primary yarn direction can lead to asymmetric shear behaviour, which may make it difficult to avoid defects during forming [5]. Here, the terms positive and negative shear refer to inplane segments of the stitch thread being in tension or compression, respectively [6], as shown in Figure 1. The influence of the intra-ply stitches on the shear resistance can be significant (for $\pm 45^{\circ}$ fabrics with pillar stitches, which are aligned at $0^{\circ}$ ) under positive shear, as the shear resistance is dominated by the tensile properties of the stitch thread. The inplane segments of the stitch are likely to fail at high tensile strains (induced by large shear 
angles), causing irreversible damage to the fabric and a reduction in the shear resistance [7]. In negative shear, NCFs tend to exhibit similar phenomena as woven fabrics when the inplane segments of the stitch are loaded in compression [1] (Figure 2). The shear stiffness is low, while the main source of shear resistance is friction between the primary yarns. This is followed by a rapid increase in shear stiffness with the onset of lateral yarn compression once the fabric locking angle is reached.

However, the fundamental difference in fabric architecture, caused by the inclusion of the stitch thread, means that the mechanisms for defect formation in NCFs are somewhat different to those in woven materials. In-plane fibre buckling [5] and out-of-plane fibre wrinkling [8] are the most common mechanisms, but fibre pull-out, stitch thread failure and inter-layer sliding have also been reported [9]. The change in fabric surface area during shear means that spaces between adjacent yarns decrease in size. Compressive stresses result in out-of-plane wrinkling [8] when lateral compaction is restricted once the shear exceeds the fabric locking angle. Most macro-scale ply wrinkles occur where large shear deformation occurs, but meso-scale bundle-level wrinkles (i.e. increase in yarn curvature between points of fixation through the stitch thread) have been reported in regions where the local shear angle was small [5].

Bias extension testing of NCF has shown that additional deformation mechanisms can also occur during fabric shear, such as fibre sliding $[9,10]$. Hemisphere forming tests indicate that fibre sliding leads to ply deterioration, where only one primary yarn direction may remain in some regions around the ply perimeter. The slip distance is dependent on the 
modulus and pre-tension of the stitching thread. If the modulus is high, the slip distance is negligible, and the NCF deformation resembles that of a woven fabric.

Few studies have been presented on the constitutive modelling of NCFs and their numerical forming simulation, despite their potential advantages in terms of manufacturing and performance over woven materials. Mesoscopic constitutive models are the most common $[6,11]$, which are related to discretisation of the fabric at the unit cell level (fibre/yarn scale). However, sufficiently fine discretisation requires significant CPU resources, which is not practical for analysing industrial-scale components. Mesoscale models are therefore limited to simulating laboratory-scale processes such as forming hemispheres. Macroscopic NCF models consider the material as a continuum based on homogenisation of several unit cells. Yu et al. [12] adapted a non-orthogonal model originally developed for woven materials at the macroscale, which successfully captured the asymmetric shear behaviour of an NCF. However, no attempt was made to correlate the macroscale behaviour to mescoscale mechanisms, in order to identify the cause of defects.

Semi-discrete approaches have also been developed [9], where the two fibre layers are modelled separately with shell elements, and 1D beam elements are used to connect them to account for the influence of the stitch. Simulation of fibre slippage can be controlled by a Coulomb friction law with a sliding threshold. However, this approach is computationally expensive as it involves additional degrees of freedom and complex contact constraints. There is currently no universal approach for modelling the deformation of bi-axial NCFs. Differences in the meso-scale architecture can significantly influence the forming behaviour and therefore the choice of modelling route. For example, the shear behaviour of a bi-axial 
NCF using a pillar stitch with high stitch stiffness may be very similar to that of a woven material, and therefore a macro-scale continuum approach may be appropriate. On the other hand, a meso-scale approach may be required for an NCF using a tricot stitch in order to capture the high levels of fibre sliding which may occur for this type of fabric.

This paper presents and validates a constitutive model for the deformation behaviour of a pillar-stitched NCF, accounting for the rotation of yarns and the direction-dependent contribution of the intra-ply stitches. The new constitutive relation is then used in a matched tool forming simulation of a hemisphere, which is validated by comparing the simulation results with experimental data. The homogenised macro-scale behaviour observed from the numerical analysis is correlated to the onset of defects during experimental preforming, allowing defect formation mechanisms to be identified.

\section{Material characterisation}

\subsection{NCF characterisation}

A bi-axial carbon fibre NCF (Hexcel FCIM359) was used for the experiments in this paper. The areal mass of the fabric is $440 \mathrm{gsm}$, and the uncompressed thickness is $0.4 \mathrm{~mm}$, as described in Table 1.

The in-plane shear behaviour of the NCF was characterised by picture frame shear tests on cruciform fabric specimens, using the methodology outlined by Harrison et al. [13]. The edge length of the testing frame, $l_{\text {frame }}$, was $145 \mathrm{~mm}$, and the length of the central region of shear deformation, $l_{\text {fabric }}$, was $110 \mathrm{~mm}$. The shear tests were carried out on a universal testing machine at a cross-head speed of $100 \mathrm{~mm} / \mathrm{min}$. The shear force, $F_{s}$, was determined 
from the force measured at the load cell, and the shear angle was calculated from the crosshead displacement, using equations presented elsewhere [14]. The normalised shear force, $F_{\text {norm }}$, can be defined as

$$
F_{\text {norm }}=\frac{F_{s} \cdot l_{\text {frame }}}{l_{\text {fabric }}^{2}}
$$

Two video cameras, positioned on either side of the test specimen, were used to record the specimen surface topography during each test and to identify the onset of defects. This qualitative information was synchronised with the corresponding shear angle calculated from the cross-head displacement. Whilst 12 tests were performed in each shear direction, not all were considered to produce average data due to the presence of outliers, which is related to common issues with misalignment of specimens in the test frame [13] .

Tests were performed on additional specimens, where stitches were removed from the NCF to isolate the contribution of the stitches on the in-plane shear behaviour from that of the primary yarns. The stitches were removed after the samples had been loaded into the picture frame. A soldering iron was used to melt the stitching thread, which avoided disturbing the underlying fibre architecture.

Figure 2 shows fundamental differences in the experimental shear behaviour between the NCF and a twill weave fabric of the same areal mass. The shear response of the woven material is symmetrical about the origin, and the normalised shear force is shown to increase steadily until the locking angle is reached. The shear behaviour of the NCF is asymmetric due to the stitch contribution. For positive shear, the normalised shear force 
rises linearly to a peak value of approximately $1 \mathrm{~N} / \mathrm{mm}$, as the stitch is loaded in tension. This peak corresponds to a shear angle of $0.5 \mathrm{rad}$, where the first stitches start to rupture. Then the shear force reduces progressively as other stitches fail successively. All stitches have failed at a shear angle of approximately $0.75 \mathrm{rad}$. There is no evident peak in the negative shear direction, as the stitches are in compression. The shape of the negative shear response for the NCF is similar to that of the woven material.

The shear curves in Figure 2 for the NCF material with and without stitches are comparable in the negative shear region ( $<0.0 \mathrm{rad})$. There is also very good agreement between the two curves for large positive shear angles (>1.0 rad), beyond the point where all stitches have failed in the stitched sample. This confirms that the large peak in the positive shear curve at approximately $0.5 \mathrm{rad}$ can be attributed to the tensile contribution of the stitches, but the shear behaviour outside this region is dominated by the rotation of the primary yarns.

\subsection{NCF material model}

A non-orthogonal constitutive model was extended here to define the asymmetric material behaviour of a bi-axial NCF, which was previously developed for woven fabrics [3, 15-17]. A user subroutine was previously developed by the authors in Abaqus/Explicit, defining the mechanical constitutive relations for woven fabrics, using a non-orthogonal relationship [3]. This approach was shown to be more accurate than an orthogonal model, as it appropriately describes the anisotropic behaviour of bi-axial materials under large shear deformation [18]. This macro-scale model can effectively capture the dominant forming mechanisms, including in-plane shear, fibre elongation (mainly related to de-crimping [19]) and inter-tow slippage. Predicted local shear angles for a woven material deviated by less than $3 \%$ from 
experimental data [3], indicating high levels of accuracy within the repeatability of the experimental test.

In the experimental results from the picture frame shear tests presented in Figure $2, a$ comparison of the stitched and unstitched curves for the NCF has been used to isolate the contribution of the intra-ply stitches. Data points in Figure 2 were selected from the stitched curve which exhibited a (Root Mean Square Error) RMSE of less than $10 \%$ compared to the unstitched curve (see blue circles in Figure 3). The polynomial function

$$
F_{\text {norm }}^{\text {yarn rotation }}=\left(29.56 \gamma_{12}^{5}-65.56 \gamma_{12}^{4}+137.06 \gamma_{12}^{3}+94.73 \gamma_{12}^{2}+112.19 \gamma_{12}\right) \mathrm{N} / \mathrm{m}(2)
$$

where $\gamma_{12}$ is the shear angle in radians, was fitted through these points (green curve in Figure 3), which is similar to the unstitched curve to model the contribution of the primary yarn rotation to the total shear resistance of the fabric. This yarn rotation component is then subtracted from the overall shear curve for the stitched NCF to determine the contribution of the stitches to the shear stiffness according to

$$
F_{\text {norm }}^{\text {stitch }}=F_{\text {norm }}-F_{\text {norm }}^{\text {yarn rotation }} \text {. }
$$

This is shown by the black diamonds in Figure 3 and can be modelled as a bilinear relationship,

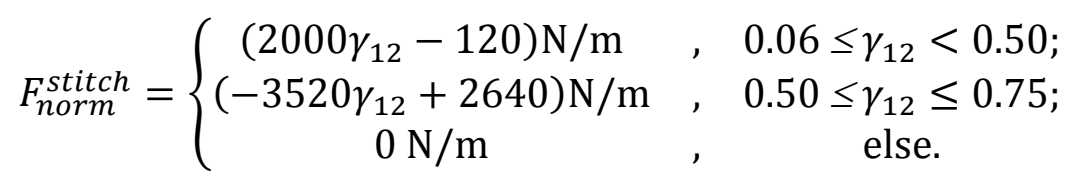


The curves indicate that there is slack in the stitches up to shear angles of approximately $0.06 \mathrm{rad}$ (i.e. approximately $1.5^{\circ}$ ), the starting point of stitch damage is $0.50 \mathrm{rad}$ (i.e. approximately $28.6^{\circ}$ ), and complete stitch failure occurs at 0.75 rad (i.e. approximately $\left.43.0^{\circ}\right)$. Thus, the stitches are active between $0.06 \mathrm{rad}$ and $0.75 \mathrm{rad}$. As shown in Figure 4, the experimental material shear curve can be reproduced by superimposing the models for the yarn rotation and stitch components, with a RMSE of less than $5 \%$.

\subsection{NCF material model validation}

Numerical tests were performed to verify that the constitutive relation used in the current user subroutine is representative of the shear behaviour of a bi-axial NCF. The material model for the stitched NCF was implemented into a finite element simulation of the picture frame shear test, for a single ply of NCF fabric. The model geometry was the same size and shape as the experimental specimens (Section 2.1). The primary yarns were parallel to the frame edges in all cases. In positive shear, the pillar stitches were aligned with the direction of crosshead travel and subjected to elongation, whilst they were perpendicular to the direction of crosshead travel for the negative shear direction. A 4-bar linkage mechanism was modelled to replicate the kinematics of the frame. The fabric was modelled using square membrane elements (M3D4R) with a size of $1 \mathrm{~mm} \times 1 \mathrm{~mm}$. A constant Young's modulus was determined using a homogenisation method [20] (138.0 GPa in each fibre direction), assuming there is no crimp in the primary yarns.

Force-displacement data from the simulation are compared against experimental picture frame shear data in Figure 5. There is good agreement (approximately $5 \%$ RMSE) between 
the simulation data and average experimental data, with the simulation curve generally falling within the bounds of the experimental test repeats.

\section{Hemisphere forming and defect formation}

In the following section, the NCF material model is implemented into an FE simulation to model the forming behaviour over a hemisphere tool. The simulation is validated by comparing the results with experimental data obtained from a laboratory-scale forming rig. A grid method is used to directly compare local shear angles and the perimeter shape from experimental samples against the simulation.

\subsection{Forming experiment}

A laboratory-scale hemisphere forming tool, integrated into a universal testing machine, was used for preforming the NCF, as shown in Figure 6 . Two $300 \mathrm{~mm} \times 300 \mathrm{~mm}$ square heated platens with central holes with $104 \mathrm{~mm}$ diameter were used as blank holders to clamp the fabric plies. Preforms were produced using two plies of bi-axial NCF (see Table 1), at $\left[0^{\circ} / 90^{\circ}\right]_{2}$, with pillar stitches at $45^{\circ}$. Two plies at the same orientation were used with a small amount (6\%wt) of reactive binder (Momentive Epikote 620) applied between the layers, in order to create a stiff preform that was suitable for measuring the fibre angles after forming. A clamping force of $1200 \mathrm{~N}$ was applied to the blank holder. A hemispherical punch with a diameter of $100 \mathrm{~mm}$ was attached to the crosshead of the machine via a $25 \mathrm{kN}$ load cell, which allowed the forming force to be monitored. A punch speed of $100 \mathrm{~mm} / \mathrm{min}$ was used to form the plies. Each forming experiment was performed at ambient temperature, before the temperature of punch and square platens was ramped to $165^{\circ} \mathrm{C}$ and held for 10 minutes to cure the binder. Experiments were conducted using punch 
displacements of both $30 \mathrm{~mm}$ (hemisphere partially formed) and $50 \mathrm{~mm}$ (hemisphere fully formed).

To quantitatively characterise fabric deformations, real-time vision systems have been described in the literature [21, 22], but their use allows defects to be identified subjectively, not in an objective and reproducible way. Other optical methods are available to compare 3D preforms to the target shape $[4,23]$, which can be used to identify deviations related to wrinkling or fibre bridging. The collected data can be mapped back onto the 3D formed shape to help identify the source of defects. Here, the visible surfaces of the NCF layers were marked with a square grid with a line spacing of $10 \mathrm{~mm}$ prior to forming (Figure 7(a)). The grid lines were aligned with the directions of the primary yarns. Grid strain analysis (GSA) [24] was used to determine the level of shear deformation in the formed hemispheres. As shown in Figure 7(b), the 3D coordinates of grid intersection points were digitised using a coordinate measuring machine (CMM), and the angles between the gridlines were calculated using GSA. A Matlab script was written to process the Cartesian coordinate data generated by the CMM, which was presented as a 3D surface plot. The coordinates of the grid intersection points were measured three times for each preform. The experimental repeatability associated with the CMM measurements was found to have a RMSE of less than $3 \%$ (Figure $7(\mathrm{c})$ ).

\subsection{D forming simulation and validation}

The hemisphere tooling was modelled using the geometry in Figure 6, with all parts considered to be rigid bodies.. A penalty contact algorithm was used to define the behaviour at all interfaces. An isotropic Coulomb friction model was adopted, assuming a constant 
friction coefficient. The tooling-fabric friction coefficient was measured to be 0.23 (sliding friction) and the fabric-fabric friction coefficient was 0.36 (plies at same orientation), according to experiments performed to ASTM D1894, ISO 8295. Displacement boundary conditions were applied to the punch to control the stroke. A force was applied to the blank holder to control fabric slippage, which was varied during the stroke from $1200 \mathrm{~N}$ to $600 \mathrm{~N}$ depending on the displacement of the punch. This accounted for a reduction in blank holder pressure related to compression of the pneumatic cylinders in the (upstroke) set-up, which was measured during the experiments.

Each $300 \mathrm{~mm} \times 300 \mathrm{~mm}$ ply was discretised into square membrane elements (M3D4R). A mesh sensitivity study was performed using the hemisphere geometry, for a punch displacement of $50 \mathrm{~mm}$. Elements with edge lengths of $1 \mathrm{~mm}, 2 \mathrm{~mm}, 3 \mathrm{~mm}, 5 \mathrm{~mm}, 10 \mathrm{~mm}$, $15 \mathrm{~mm}$ and $20 \mathrm{~mm}$ were investigated. A box-and-whisker plot of shear angle is presented in Figure 11 for each element size. The median, $25^{\text {th }}$ percentile and $75^{\text {th }}$ percentile are all similar for each data set, which indicates that all element edge lengths produce a representative distribution of shear angles over the surface of the hemisphere. However, the maximum positive shear angle increases by more than $10 \%$ from $30.6^{\circ}$ to $34.5^{\circ}$ when the element length decreases from $15 \mathrm{~mm}$ to $10 \mathrm{~mm}$. The maximum shear angle converges towards a value of $36^{\circ}$ as the element size approaches $1 \mathrm{~mm}$. A $1 \mathrm{~mm}$ element size has been adopted throughout this work.

The simulation results for two punch displacements are compared to the average of the experimentally determined grid coordinates (from 3 repeats) in Figure 8 . The simulated grid intersections are a close match to the measured points, which indicates that the model is 
able to predict the forming behaviour of the NCF with good accuracy. The error for the 50 $\mathrm{mm}$ punch displacement is larger than for the $30 \mathrm{~mm}$ displacement, i.e. the accuracy reduces with increasing punch displacement. The largest differences between measured and simulated coordinates at grid points $(1.79 \mathrm{~mm}$ for the $30 \mathrm{~mm}$ case and $2.87 \mathrm{~mm}$ for the $50 \mathrm{~mm}$ case) occur at points in the positive shear regions (i.e. top-right and bottom-left quadrant in Fig. 8), which exhibit the highest degree of wrinkling. These regions also show the largest difference in data measured for different specimens (while the reproducibility of measurements for each specimen is high, see Figure 7) using the GSA approach, as the tension of the stitches and the initial orientation of the primary yarns may vary, affecting the level and position of local wrinkling. In addition, the membrane model used here neglects the change in fabric thickness and out-of-plane bending stresses. Therefore, the accuracy of the predicted local response of the fabric may be compromised at the finite element scale, but the global response is still representative.

The shear angle at each of the 961 grid intersections was calculated from both the experimentally determined and predicted coordinates using GSA. As shown in Figure 9, the overall shear angle distributions from the simulation are a good representation of the corresponding experimental data. The difference in local shear angle is less than $5^{\circ}$. More accurate prediction of shear deformation is obtained for the case using a smaller punch displacement $(30 \mathrm{~mm})$, since there are fewer wrinkling defects compared to the larger punch displacement $(50 \mathrm{~mm})$. The largest differences in shear angle are limited to relatively small areas around the wrinkle regions and near the edge of the blank, which are a result of the density of the grid used on the experimental samples. 
Further analysis was conducted by comparing probability density curves of the experimentally determined and predicted shear angle distributions for two punch displacements (Figure 10). The corresponding pairs of curves show good agreement with each other. The difference between curves derived from the experiment and simulation for the $30 \mathrm{~mm}$ punch displacement is small (RMSE $<5 \%$ ). The peak shear angles in the negative and positive regions are predicted with high accuracy. There is some uncertainty in the range of small shear angles (i.e. around zero degree), which may be related to the very small difference in shear angles between neighbouring elements at the beginning of the simulation. The difference increases for the $50 \mathrm{~mm}$ punch displacement due to the instabilities caused by the increase in wrinkling defects, where it is approximately $7 \%$ (RMSE). The largest discrepancy appears in the range of small shear angles, which is similar to the $30 \mathrm{~mm}$ case.

\subsection{Defect formation in bi-axial NCF}

Figure 12 confirms that the level of intra-ply slip is negligible in the matched tool forming process, as the difference in grid strains between the convex and concave sides of the formed hemisphere is less than $3 \%$. This is within the systematic uncertainty for the coordinate measurement reported in Figure 7. There was also no significant difference in grid deformation (including perimeter shape), and no visible sign of ply deterioration. This supports the decision to use a macro-scale non-orthogonal constitutive model to describe the forming behaviour of this bi-axial NCF.

The wrinkling strain, which gives rise to local yarn deformation, was defined as the negative strain (i.e. compressive strain) along each primary fibre direction $\left(\varepsilon_{f 11}, \varepsilon_{f 22}\right)$. Unlike for 
woven fabrics, only one principal yarn orientation is visible from each side of a bi-axial NCF ply. Thus, wrinkling defects may appear differently on the concave and convex side of the hemisphere. Here, yarns on the convex side were initially orientated at $0^{\circ}$ (aligned with the $x$-axis in Figure 12 ), and therefore wrinkling defects are indicated by the wrinkling strain along the first fibre orientation (i.e. $\varepsilon_{f 11}$ ). The fibres on the concave side of the hemisphere were orientated at $90^{\circ}$ (aligned with the y-axis in Figure 12) before preforming, and local wrinkling on this side is highlighted by the wrinkling strain along the second fibre orientation (i.e. $\left.\varepsilon_{f 11}\right)$. Figure 13 confirms that wrinkling defects occur at different positions on different sides of the bi-axial NCF ply.

Comparing the shear angle distributions (Figure 9) and wrinkling strain distributions along both fibre orientations (Figure 13) confirms that the majority of wrinkling defects occur within the highly sheared regions. There is a positive correlation between the shear deformation and the wrinkling strain, indicating the likelihood of wrinkles. This has been previously observed by the authors during fabric forming optimisation studies $[3,15,17]$. Two different kinds of wrinkling defects can be observed from Figure 13. Out-of-plane ply folds can be observed at the macro-scale, and in-plane bundle waviness can be observed at the meso-scale. These are easily distinguishable and provide an effective way to trace the cause of the defects, by comparing these observations against the simulations.

In order to determine the wrinkle onset point and the cause, hemispheres were preformed at increasing punch displacement $(10 \mathrm{~mm}, 30 \mathrm{~mm}$ and $50 \mathrm{~mm})$ as shown in Figure 14. Out-ofplane macro-scale wrinkling, which results in an uneven surface, can be observed mainly where positive shear dominates (Figure 14(a)). Since intra-ply stitches provide additional 
constraints on yarn rotation in these regions, shear locking occurs at smaller shear angles than in regions with negative shear. Compressive in-plane strain then results in out-of-plane wrinkling as shown in Figure 14(a), which can be observed on both sides of the ply. Figure 9 indicates that some shear angles in the positive shear regions (for both $30 \mathrm{~mm}$ and $50 \mathrm{~mm}$ cases) have exceeded a shear angle of $0.5 \mathrm{rad}$ (i.e. $28.6^{\circ}$ ) when individual intra-ply stitches start to fail (Figure 4). This indicates that the predicted wrinkling defects around this area (Figure 14(a)) can be attributed to excessive shearing, which is in agreement with the experiment.

Meso-scale wrinkling occurs mainly in the negatively sheared region of the part (Figure 14(b)). The surface roughness is unaffected, but in-plane fibre waviness is visible on the surface of the ply. The primary yarns can rotate more freely in-plane under negative shear, as the shear resistance is derived from in-plane friction between yarns and lateral yarn compression. Therefore in-plane fibre buckling occurs. As shown in Figure 9, the maximum absolute shear angles in the negative shear region are less than $60^{\circ}$ for all cases. Visual observation of the picture frame shear tests (Section 2.1) has indicated that no defects occur in negative shear within this range of angles. Hence, it can be concluded that defect formation in these regions is not related to excessive shearing.

According to Figure 13 and Figure 14, wrinkling occurs at a wrinkling strain of approximately -0.03 , indicating some resistance to fibre buckling at lower strains. Defect formation can therefore be identified with good accuracy from simulation results, by combined evaluation of the shear angle and wrinkling strain distributions. The latter predicts potential areas for 
meso- and macro-scale wrinkling, without distinguishing type, whilst the former indicates defects related to macro-scale wrinkling only.

\section{Conclusions}

Intra-ply stitches, which are used to assemble two layers of yarns to produce one ply of biaxial NCF, can introduce asymmetry to the fabric shear behaviour. In particular, pillar stitches in bi-axial NCFs make a significant directional contribution to the NCF shear resistance and affect the defect formation mechanisms in preforming. A constitutive model was developed and implemented in FE simulations to predict this behaviour, using a nonorthogonal framework to track the orientation of primary yarns at the macro-scale. Picture frame shear testing was performed to capture the asymmetric shear behaviour of the biaxial NCF, providing input data in the form of shear resistance as a function of shear angle.

In order to identify the mechanisms of defect formation in detail, the shear resistance was decomposed into two constituents, primary yarn rotation and tension in the stitching thread. The fibre rotation was described using a low-order polynomial, and the stitch behaviour was represented by a bilinear relation. A deviation of approximately $5 \%$ was observed between the simulation and experimental data when the model was applied to a planar shear case. The simulation curve generally falls within the bounds of the experimental data for 12 experimental repeats.

A detailed study was conducted to investigate the onset and propagation of defects for a biaxial NCF with a pillar stitch, formed over a hemisphere tool. Grid strain analysis was 
employed to validate the simulation, and it was found that both sets of local shear angles differed by less than $5^{\circ}$ at maximum punch displacement.

The model predicts different local distributions of forming defects on both sides of a NCF ply. Two types of defect mechanism were identified in hemisphere forming. Comparisons with the simulation results indicate that one is caused by excessive shear, inducing out-ofplane wrinkling (macro-scale wrinkling), another is caused by fibre compression, inducing inplane wrinkling (meso-scale wrinkling). By correlating the local distributions of shear angle and wrinkling strain, it is possible to determine the wrinkling mode and confirm the corresponding defect mechanism. Results show that the predicted wrinkling position and defect mechanism correlate well with the experiments, which further supports the validity of the NCF material model and the FE forming process simulation.

\section{Acknowledgements}

The work presented in this paper was completed as part of the "Affordable Lightweighting Through Pre-form Automation" (ALPA) project. The authors gratefully acknowledge the financial support of Innovate UK (Project Ref. 101879).

\section{References}

[1] Lomov, S.V., M. Barburski, T. Stoilova, I. Verpoest, R. Akkerman, R. Loendersloot, and R.H.W.t. Thije, Carbon composites based on multiaxial multiply stitched preforms. Part 3: Biaxial tension, picture frame and compression tests of the preforms. Composites Part A: Applied Science and Manufacturing, 2005. 36(9): p. 1188-1206.

[2] Tanaka, K., R. Ushiyama, T. Katayama, S. Enoki, and H. Sakamoto. Formability evaluation of carbon fiber NCF by a non-contact 3D strain measurement system and the effects of blank folder force on its formability. in WITPress. 2014. 
[3] Chen, S., A. Endruweit, L. Harper, and N. Warrior, Inter-ply stitching optimisation of highly drapeable multi-ply preforms. Composites Part A: Applied Science and Manufacturing, 2015. 71: p. 144-156.

[4] Margossian, A., S. Bel, J. Balvers, D. Leutz, R. Freitas, and R. Hinterhoelzl, Finite element forming simulation of locally stitched non-crimp fabrics. Composites Part A: Applied Science and Manufacturing, 2014. 61: p. 152-162.

[5] Lee, J.S., S.J. Hong, W.-R. Yu, and T.J. Kang, The effect of blank holder force on the stamp forming behavior of non-crimp fabric with a chain stitch. Composites Science and Technology, 2007. 67(3-4): p. 357-366.

[6] Creech, G. and A. Pickett, Meso-modelling of non-crimp fabric composites for coupled drape and failure analysis. Journal of materials science, 2006. 41(20): p. 6725-6736.

[7] Dumont, F., C. Weimer, D. Soulat, J. Launay, S. Chatel, and S. Maison-Le-Poec, Composites preforms simulations for helicopters parts. International Journal of Material Forming, 2008. 1(1): p. 847-850.

[8] Yin, H., X. Peng, T. Du, and Z. Guo, Draping of plain woven carbon fabrics over a doublecurvature mold. Composites Science and Technology, 2014. 92: p. 64-69.

[9] Bel, S., N. Hamila, P. Boisse, and F. Dumont, Finite element model for NCF composite reinforcement preforming: Importance of inter-ply sliding. Composites Part A: Applied Science and Manufacturing, 2012. 43(12): p. 2269-2277.

[10] Bel, S., P. Boisse, and F. Dumont, Analyses of the deformation mechanisms of non-crimp fabric composite reinforcements during preforming. Applied Composite Materials, 2012. 19(3-4): p. 513-528.

[11] Sirtautas, J., A.K. Pickett, and P. Lépicier, A mesoscopic model for coupled drape-infusion simulation of biaxial Non-Crimp Fabric. Composites Part B: Engineering, 2013. 47: p. 48-57.

[12] Yu, W.-R., P. Harrison, and A. Long, Finite element forming simulation for non-crimp fabrics using a non-orthogonal constitutive equation. Composites Part A: Applied Science and Manufacturing, 2005. 36(8): p. 1079-1093.

[13] Harrison, P., M. Clifford, and A. Long, Shear characterisation of viscous woven textile composites: a comparison between picture frame and bias extension experiments. Composites Science and Technology, 2004. 64(10): p. 1453-1465.

[14] Cao, J., R. Akkerman, P. Boisse, J. Chen, H. Cheng, E. De Graaf, J. Gorczyca, P. Harrison, G. Hivet, and J. Launay, Characterization of mechanical behavior of woven fabrics: experimental methods and benchmark results. Composites Part A: Applied Science and Manufacturing, 2008. 39(6): p. 1037-1053.

[15] Chen, S., L. Harper, A. Endruweit, and N. Warrior, Formability optimisation of fabric preforms by controlling material draw-in through in-plane constraints. Composites Part A: Applied Science and Manufacturing, 2015. 76: p. 10-19.

[16] Chen, S., A. Endruweit, L. Harper, and N. Warrior, Forming simulations of multi-layered woven preforms assembled with stitch yarns, in 16th European Conference on Composite Materials. 2014: Seville Spain.

[17] Chen, S., L.T. Harper, A. Endruweit, and N.A. Warrior, Optimisation of forming process for highly drapeable fabrics, in 20th International Conference on Composite Materials. 2015: Copenhagen Denmark.

[18] Peng, X. and F. Ding, Validation of a non-orthogonal constitutive model for woven composite fabrics via hemispherical stamping simulation. Composites Part A: Applied Science and Manufacturing, 2011. 42(4): p. 400-407.

[19] Cavallaro, P.V., A.M. Sadegh, and C.J. Quigley, Decrimping behavior of uncoated plain-woven fabrics subjected to combined biaxial tension and shear stresses. Textile Research Journal, 2007. 77(6): p. 403-416.

[20] Peng, X. and J. Cao, A dual homogenization and finite element approach for material characterization of textile composites. Composites Part B: Engineering, 2002. 33(1): p. 45-56. 
[21] Schmitt, R., T. Furtjes, B. Abbas, P. Abel, W. Kimmelmann, P. Kosse, and A. Buratti, Real-time machine vision system for an automated quality monitoring in mass production of multiaxial non-crimp fabrics. IFAC-PapersOnLine, 2015. 48(3): p. 2393-2398.

[22] Strauf Amabile, M., V. Eckers, and T. Gries, Draping of non-crimp fabrics for fibre reinforced composites. International Journal of Material Forming, 2010. 3(1): p. 647-650.

[23] Krieger, H., A. Schnabel, L. Appel, and T. Gries, Experimental setup to validate textile material models for drape simulation. Key Engineering Materials, 2013. 554-557: p. 456-464.

[24] Martin, T., G. Christie, and D. Bhattacharyya, Grid strain analysis and its application in composite sheet forming, in Composite Sheet Forming, D. Bhattacharyya, Editor. 1997, Elsevier. p. 217-245. 


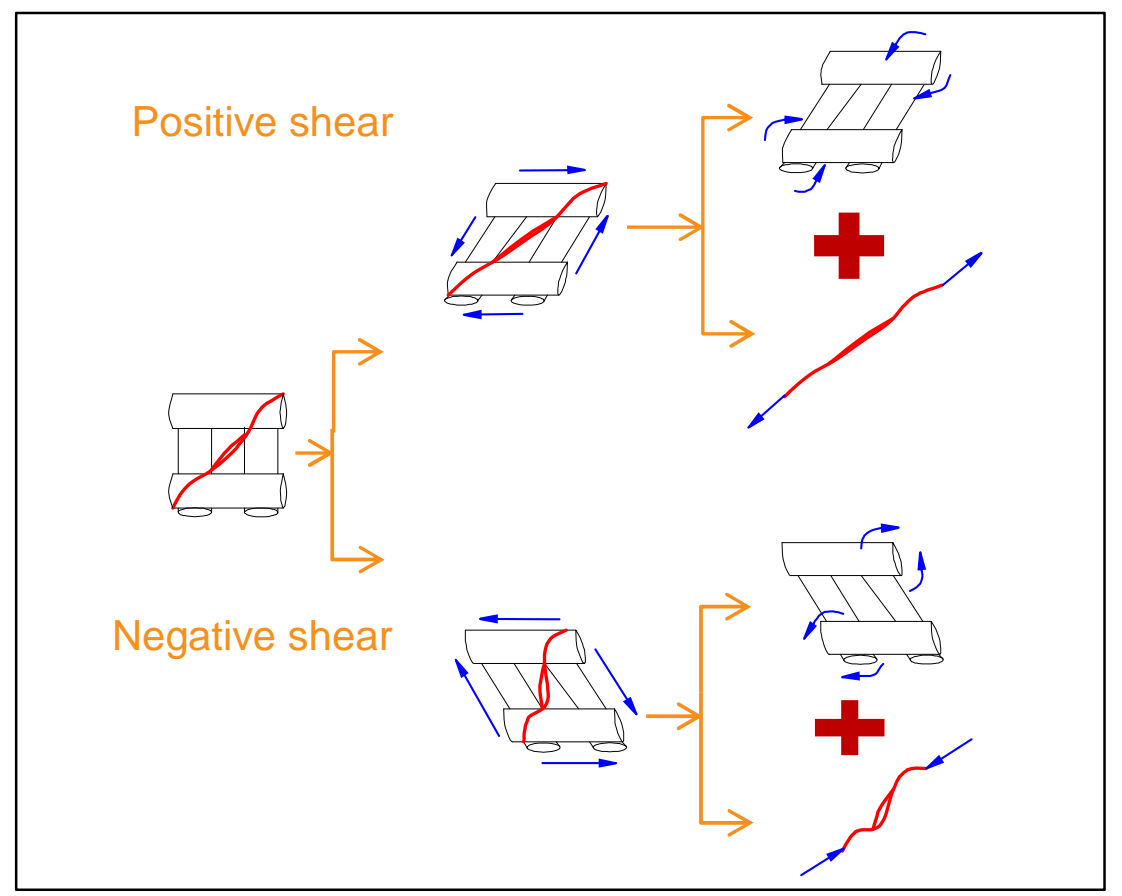

Figure 1: Schematic showing the difference between positive and negative shear. In this example, the primary yarns are at $0^{\circ} / 90^{\circ}$ and the in-plane segment of the stitching thread is at $45^{\circ}$.

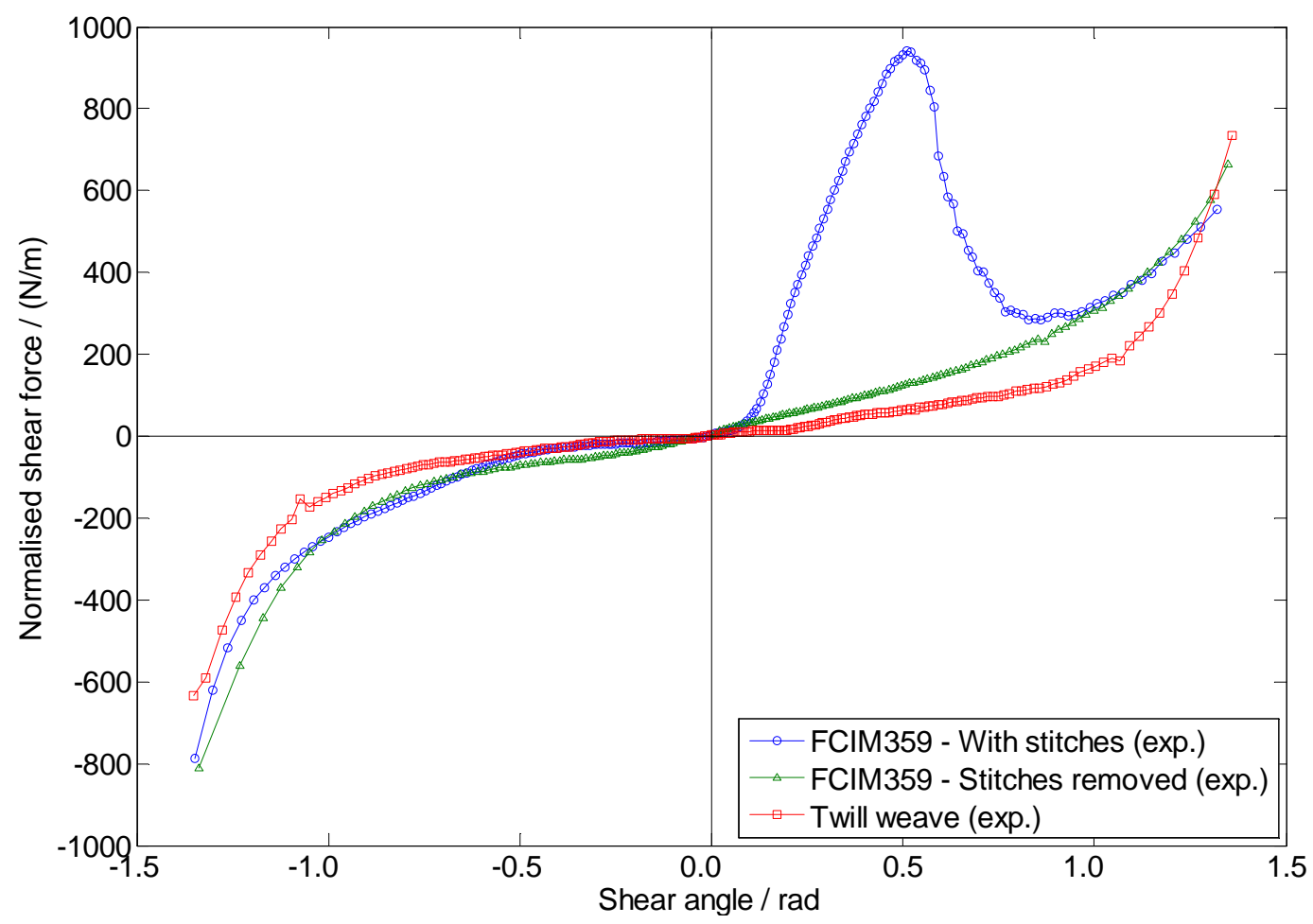

Figure 2: Experimental picture frame shear results (average values) for FCIM359 bi-axial NCF. Testing was performed in two directions, placing the stitch yarn in tension (positive shear) and compression (negative shear). Stitches were fully removed in some samples. Results for a $400 \mathrm{gsm}$ twill weave fabric are shown for comparison. 


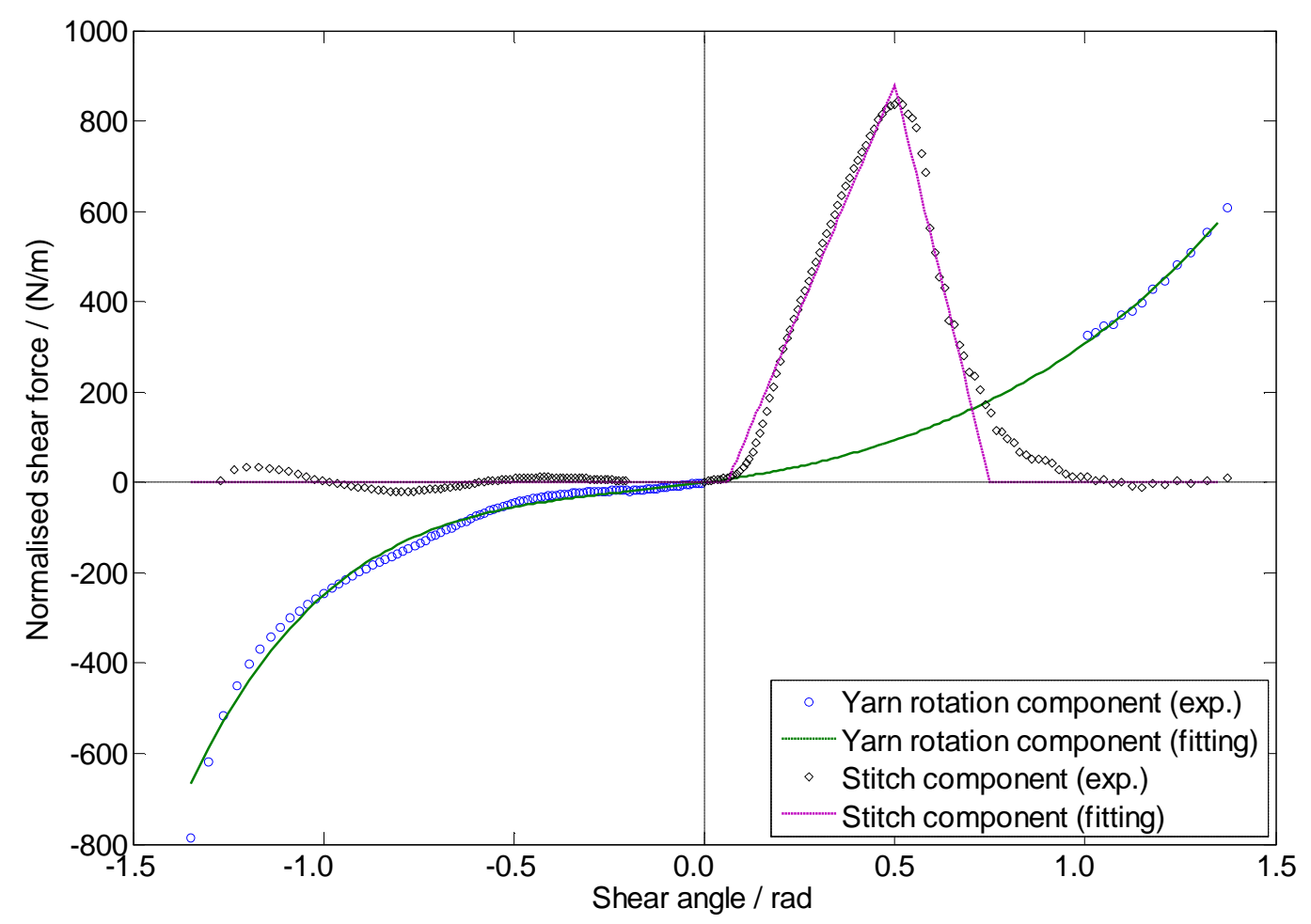

Figure 3: Shear resistance curve of FCIM359 (taken from Figure 2) separated into two components: Primary yarn rotation and stitch contribution.

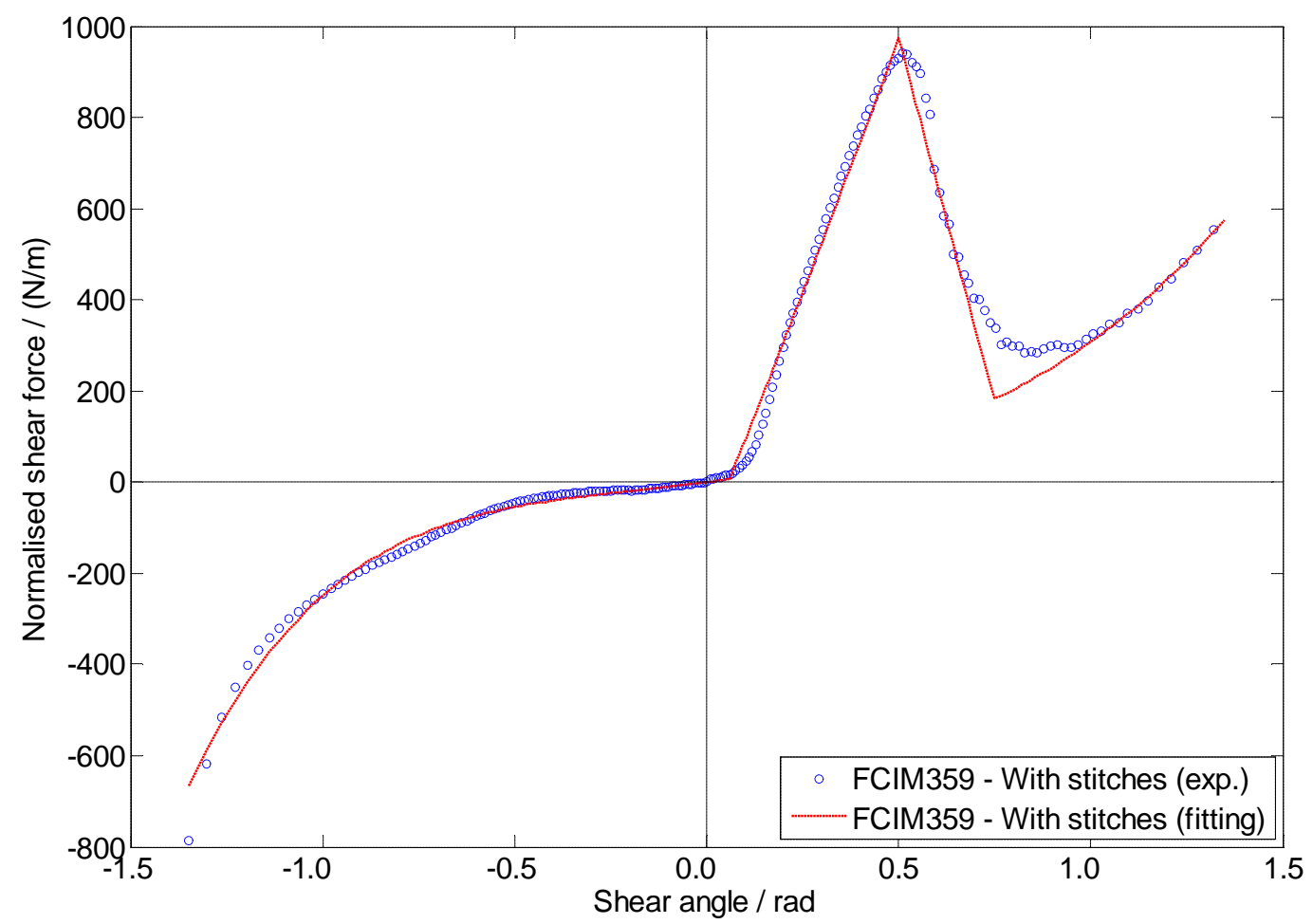

Figure 4: Summary of the shear resistance curve for fully stitched FCIM359 bi-axial NCF. Red line shows the numerical fit. 


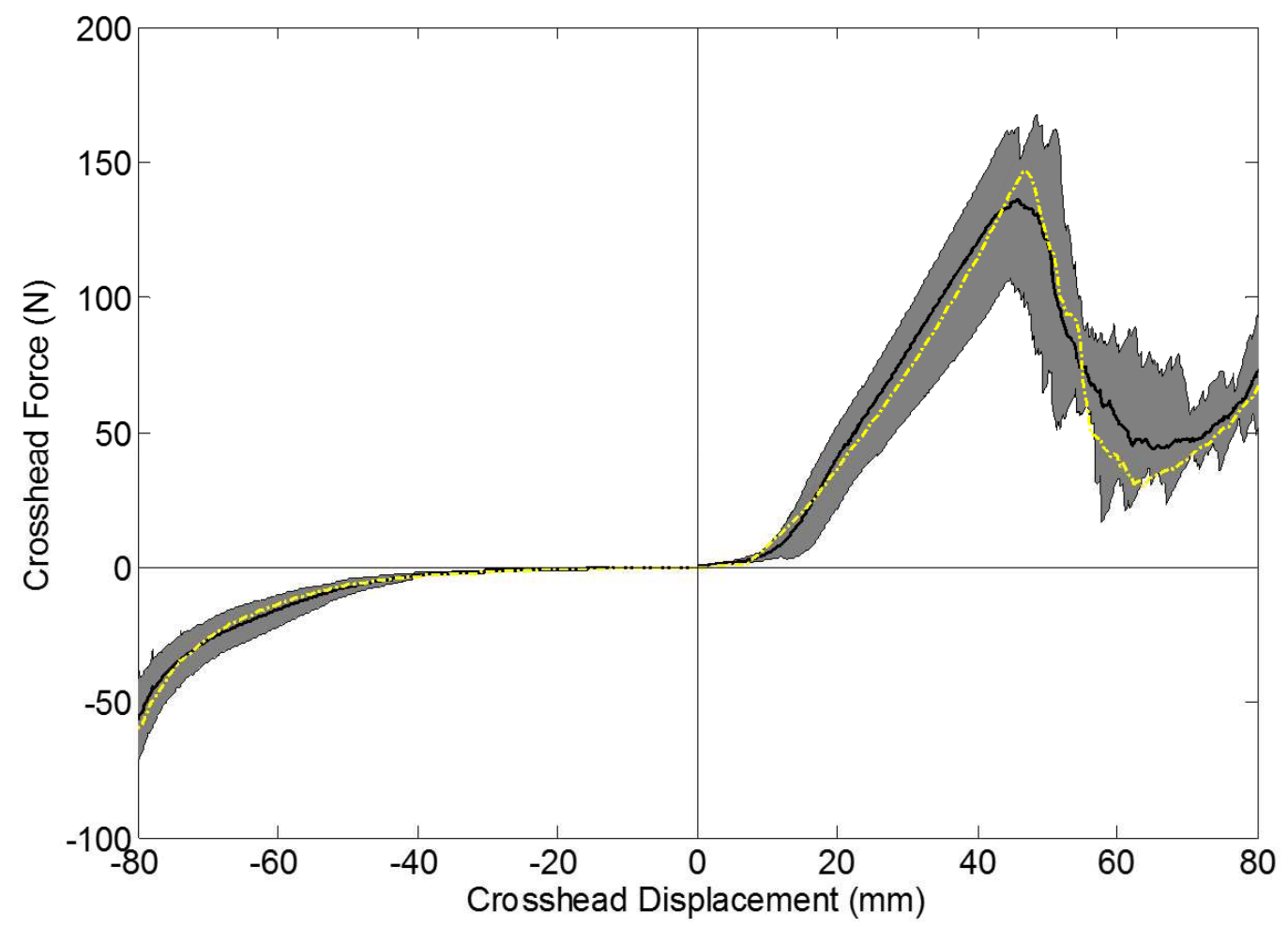

Figure 5: Comparison of shear resistance curves from picture frame shear experiments (black: average; grey: range of results from 7 tests in the negative shear direction and 8 tests in the positive direction); yellow: simulations.

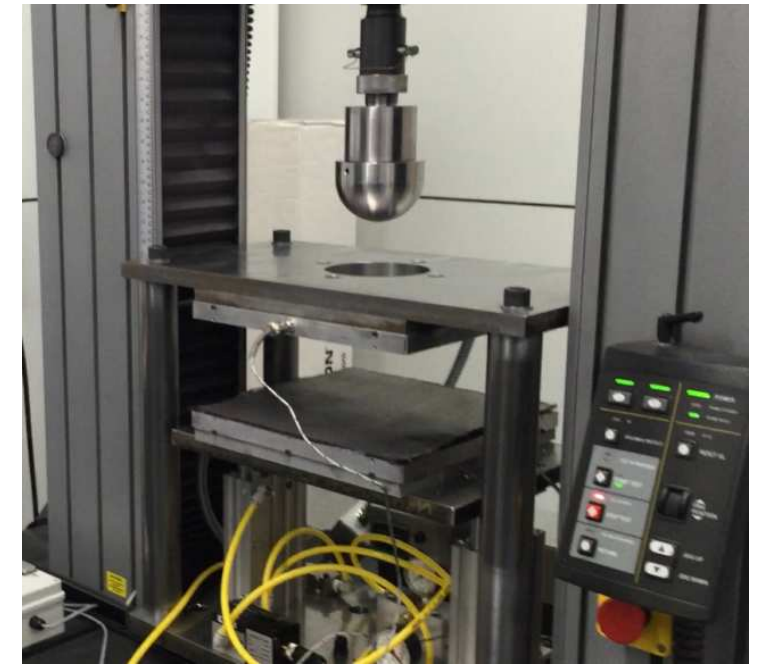

(a) Tool setup

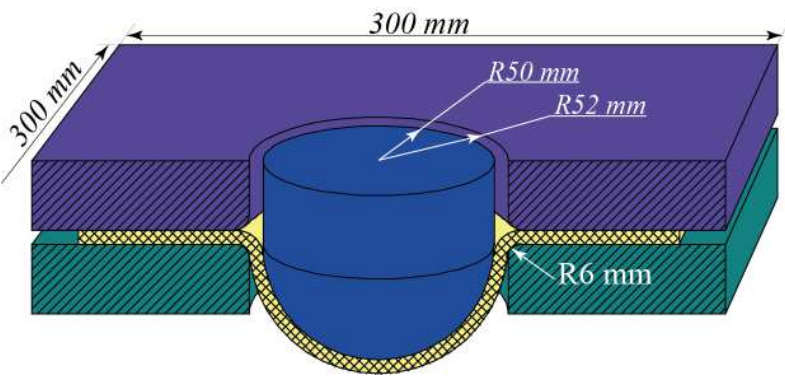

(b) Dimensions

Figure 6: Hemisphere forming tool. 


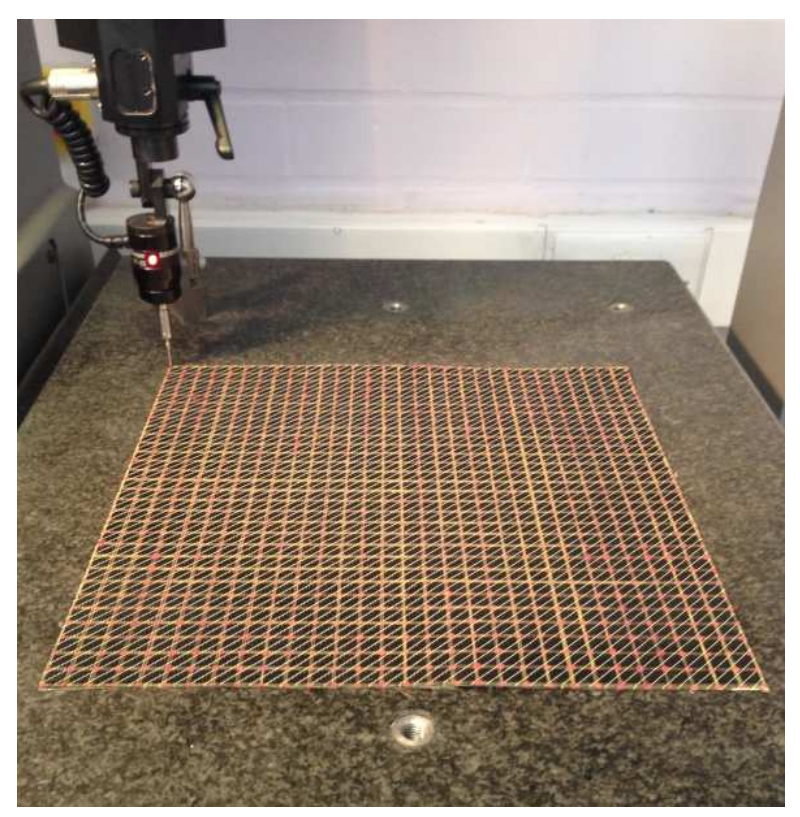

(a) Undeformed fabric

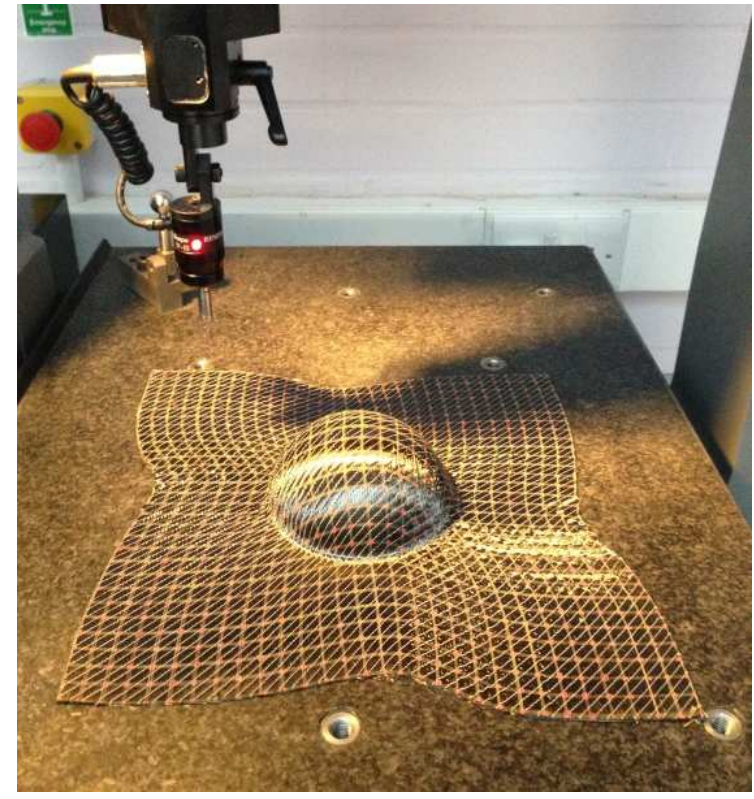

(b) Deformed fabric

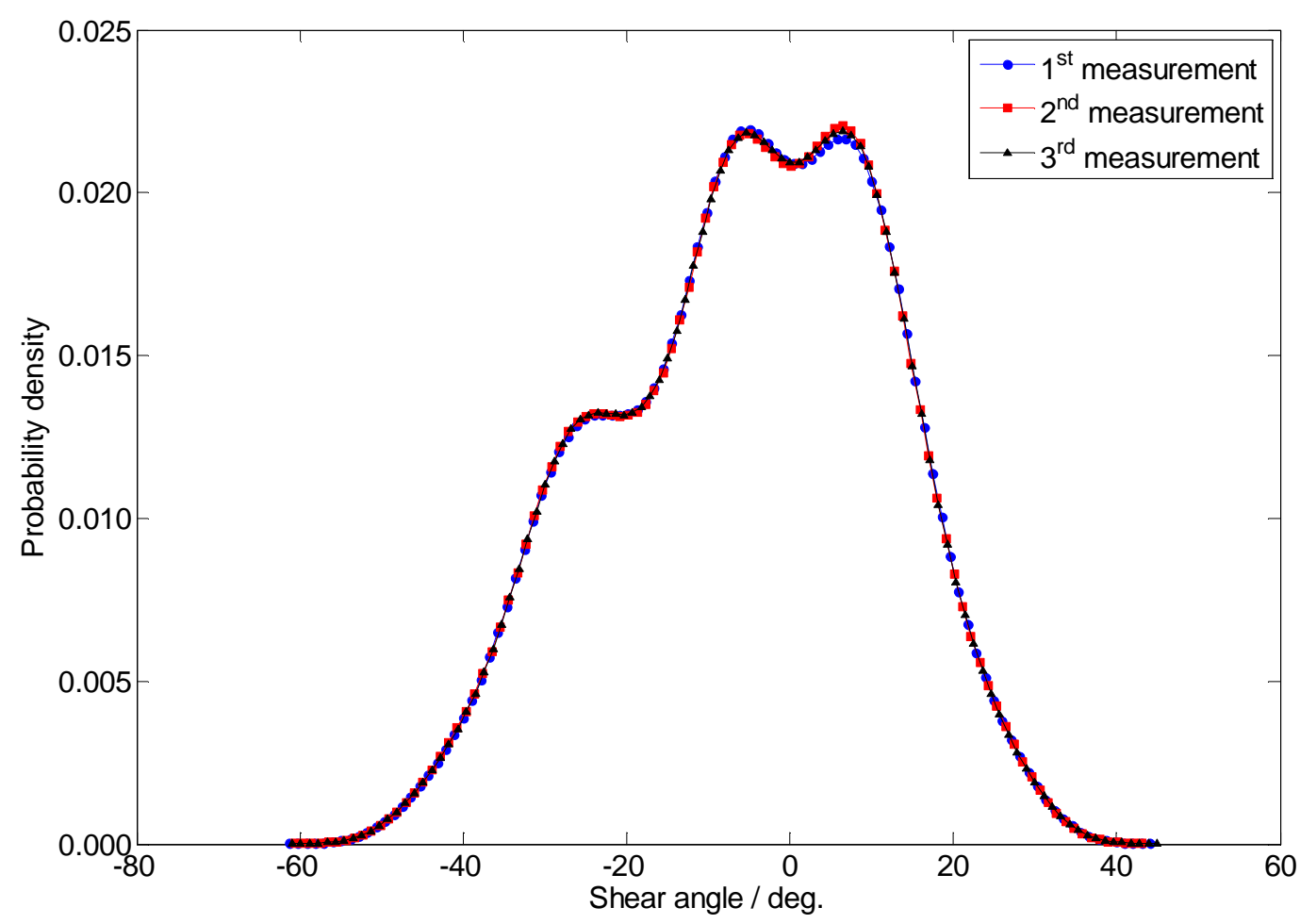

(c) Probability density of shear angles measured three times independently on a preform at a displacement of the hemispherical punch of $50 \mathrm{~mm}$

Figure 7: Grid measurement for NCF preforms using a coordinate measuring machine (CMM). 


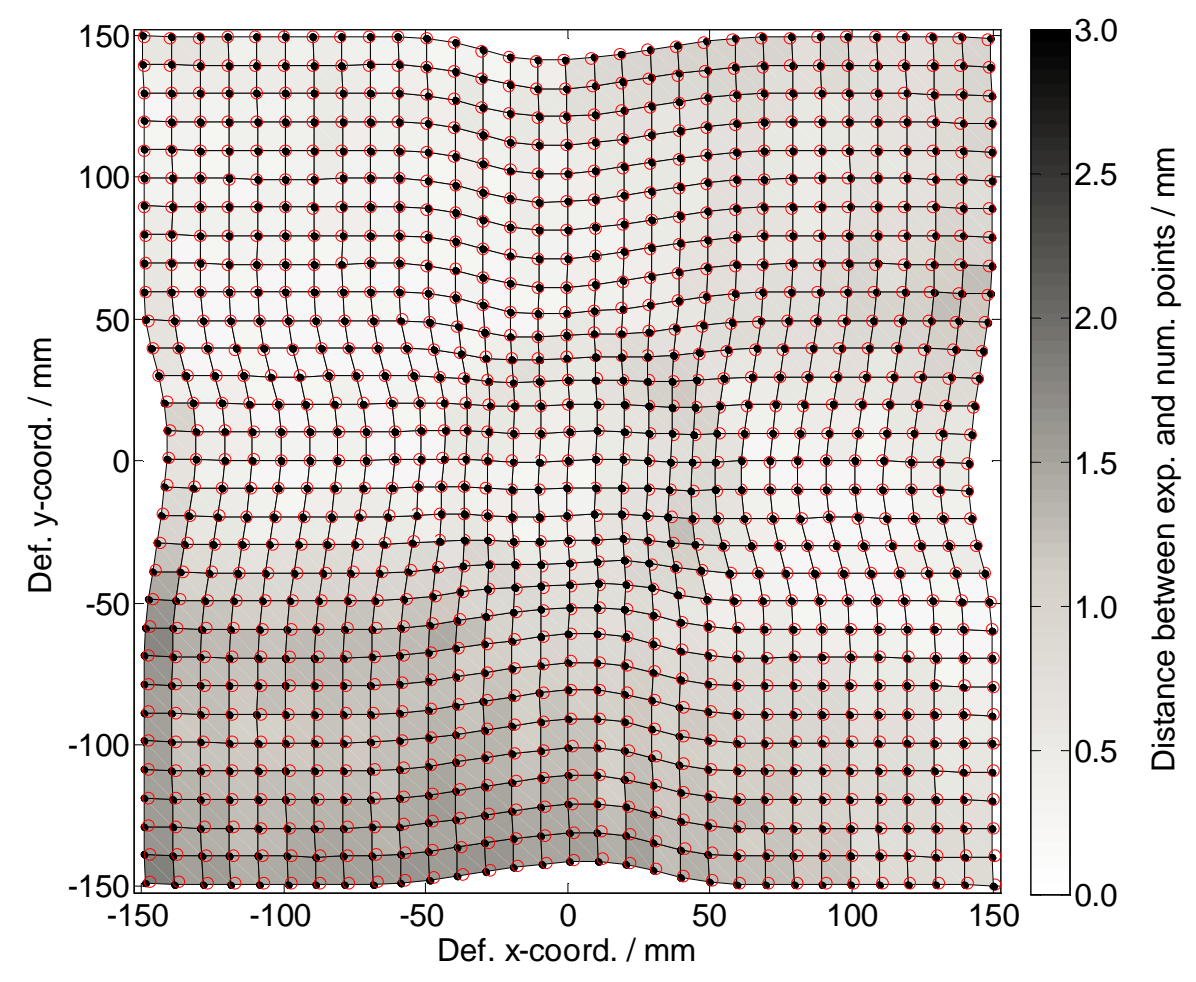

(a) $30 \mathrm{~mm}$ punch displacement

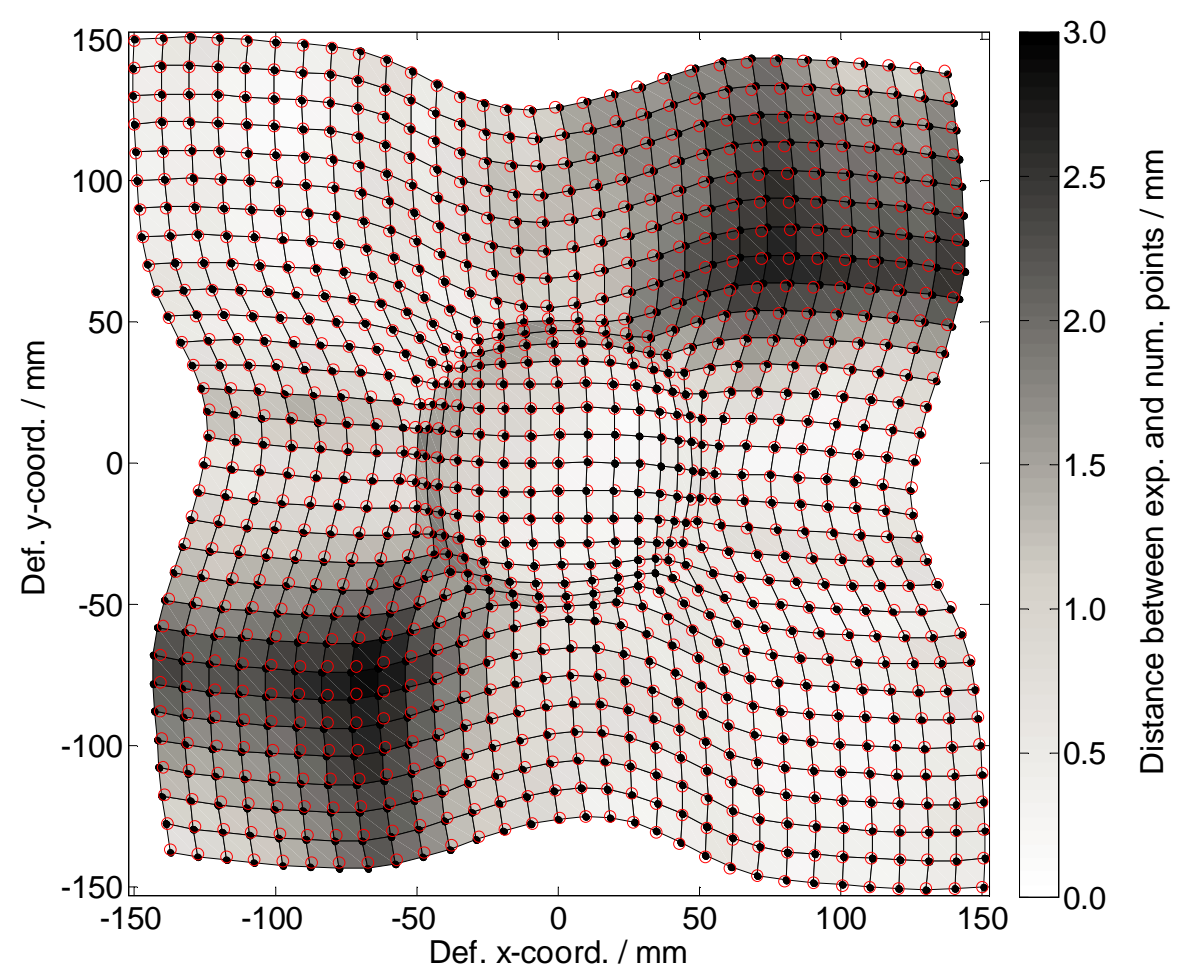

(b) $50 \mathrm{~mm}$ punch displacement

Figure 8: Comparison of experimental (red circles) and numerical (black solid dots) grids. The contour shows the distance between the grid intersections obtained from experiment and simulation. 

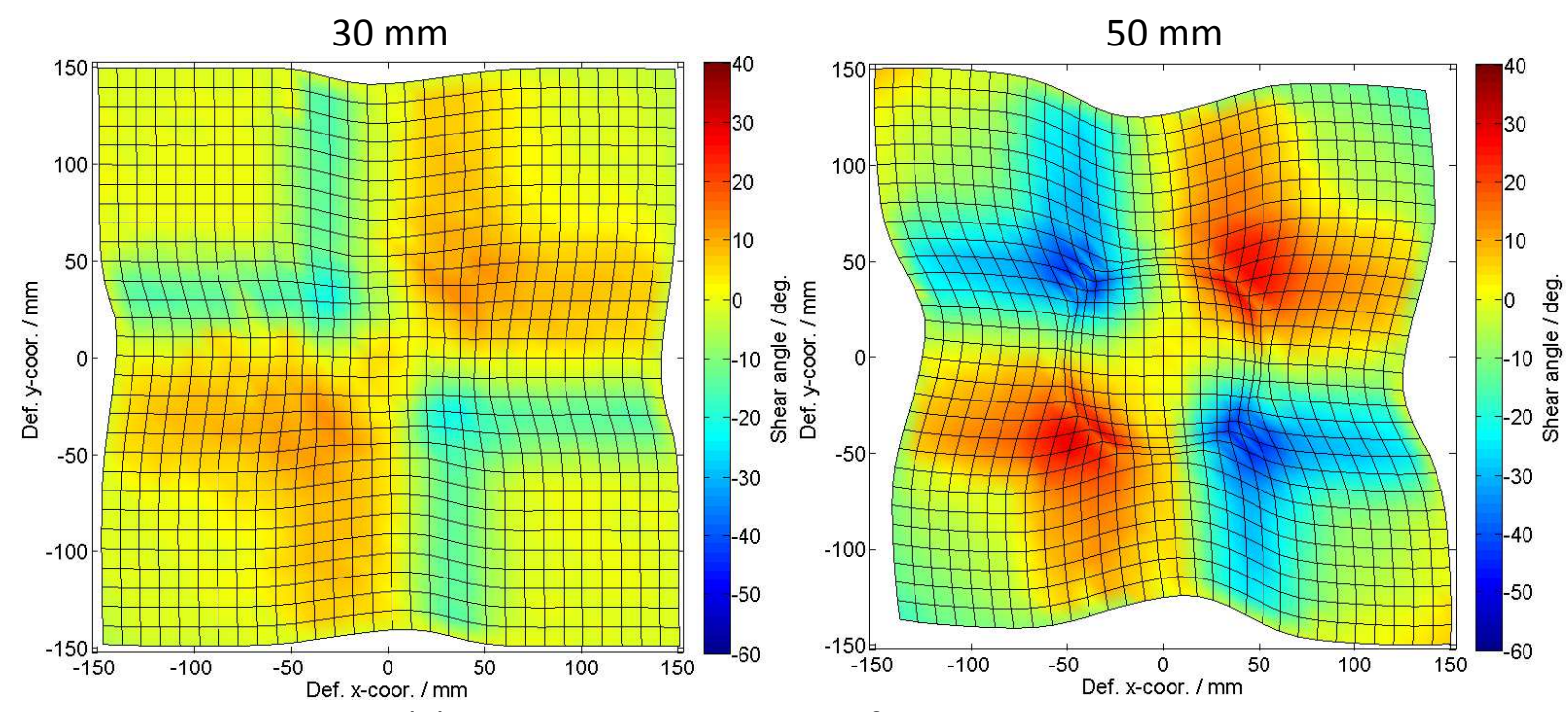

(a) Shear angle distribution from experiment

$30 \mathrm{~mm}$

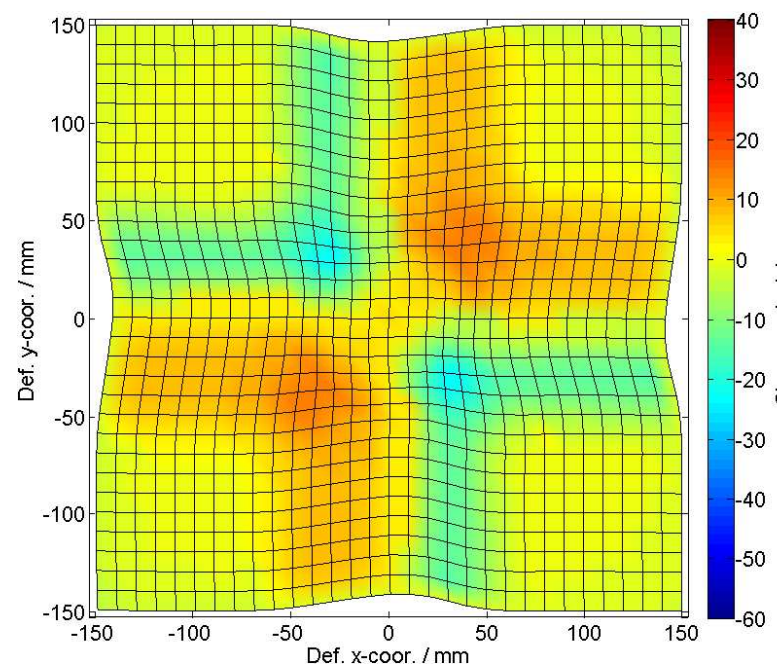

(b) Shear angle distribution from simulation
$50 \mathrm{~mm}$

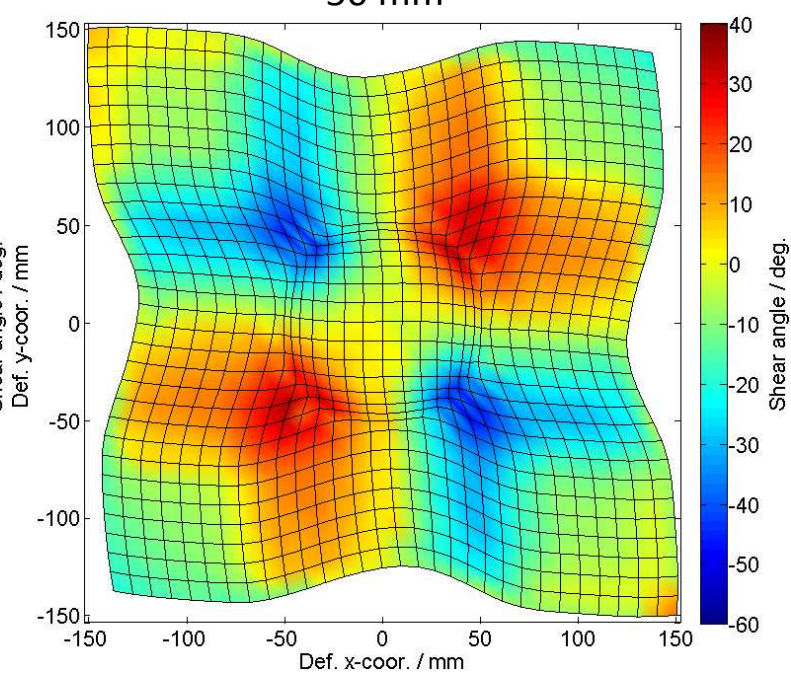

$50 \mathrm{~mm}$

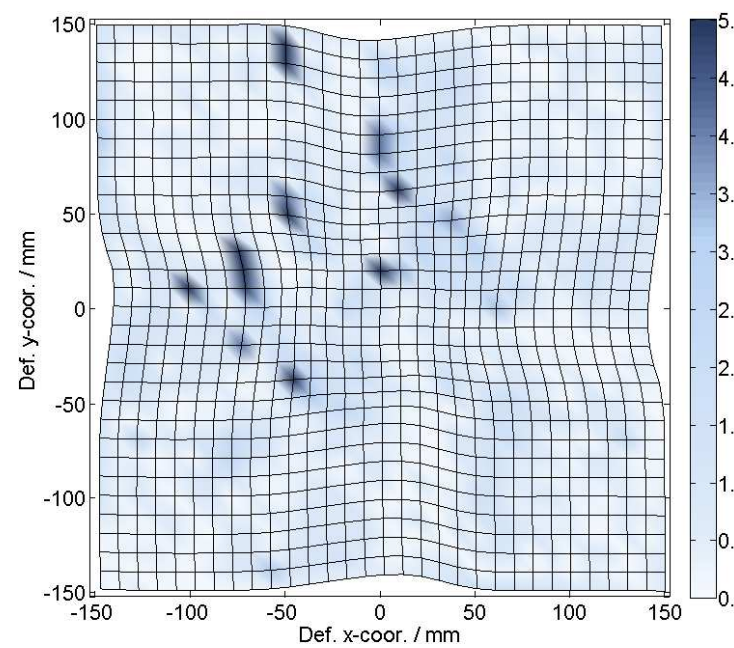

(c) Difference of shear angle distributions from simulation and experiment

Figure 9: Comparison of shear angle distributions from experiment and simulation determined using GSA. 


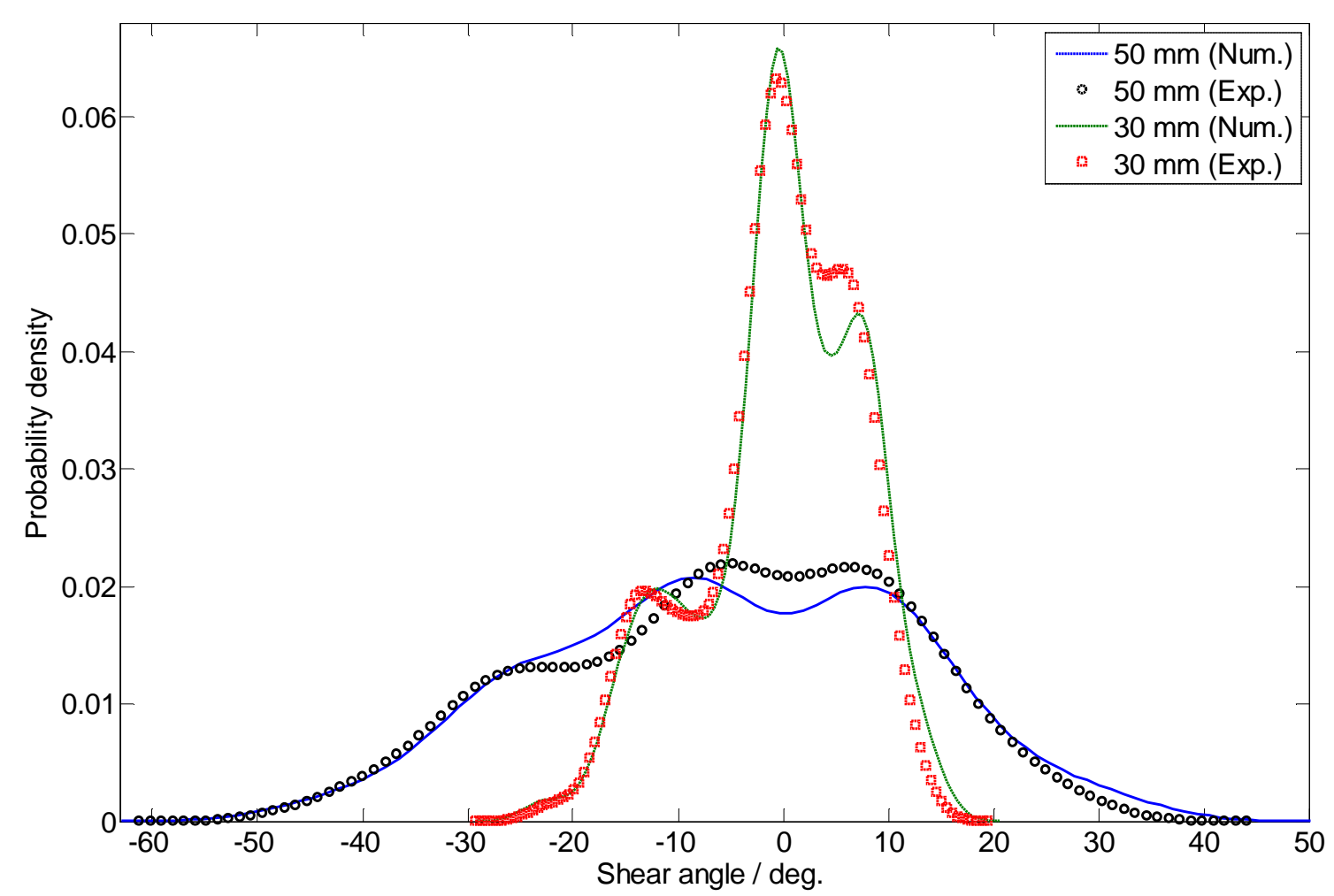

Figure 10: Probability density of shear angle from simulation and experiment using punch displacements of $30 \mathrm{~mm}$ and $50 \mathrm{~mm}$.

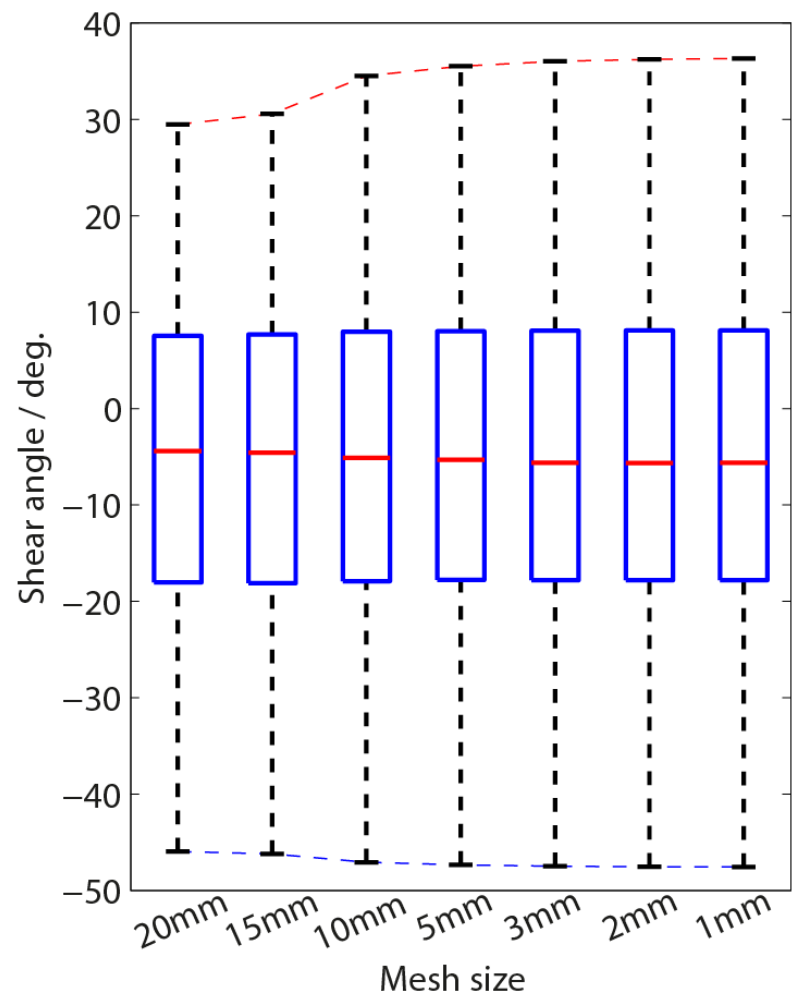

Figure 11: Box-and whisker plots for shear angles from FE simulations using different mesh sizes. The central red line represents the median, the horizontal box edges represent the $25^{\text {th }}$ and $75^{\text {th }}$ percentiles and the dotted lines represent the maximum and minimum values in each case. 


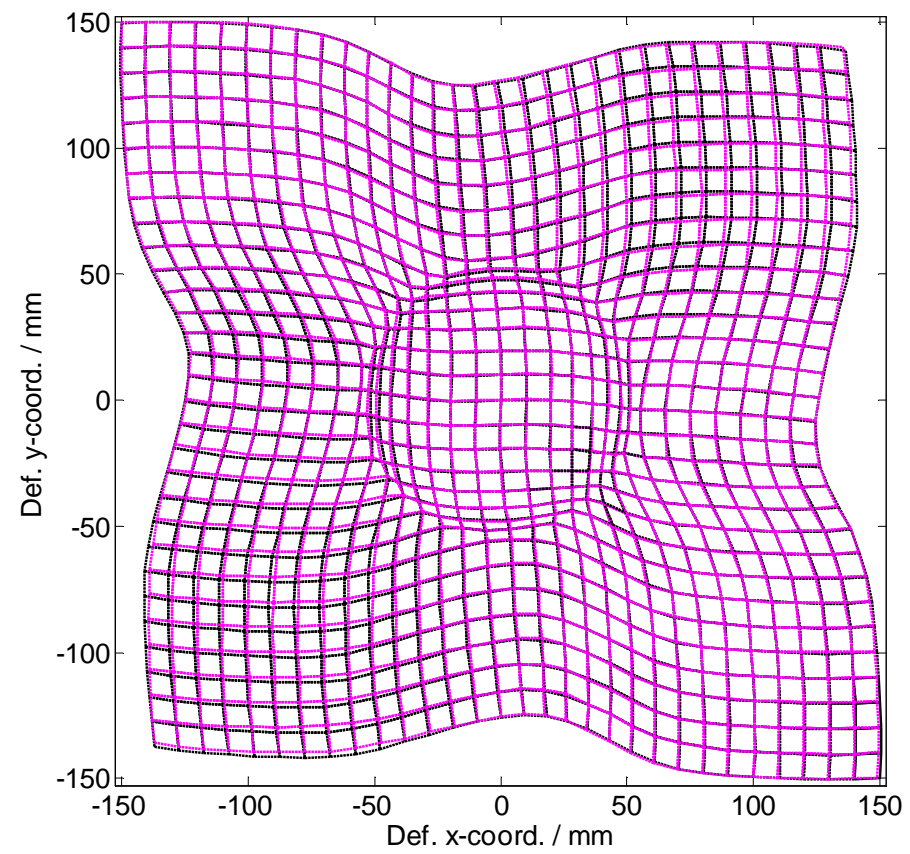

Figure 12: Comparison of grids measured from both sides of preforms at a punch displacement of $50 \mathrm{~mm}$. Black solid grid: measured on hemisphere concave side (yarns initially along $90^{\circ}$ ); purple dashed grid: measured on hemisphere convex side (yarns initially along $0^{\circ}$ ). 

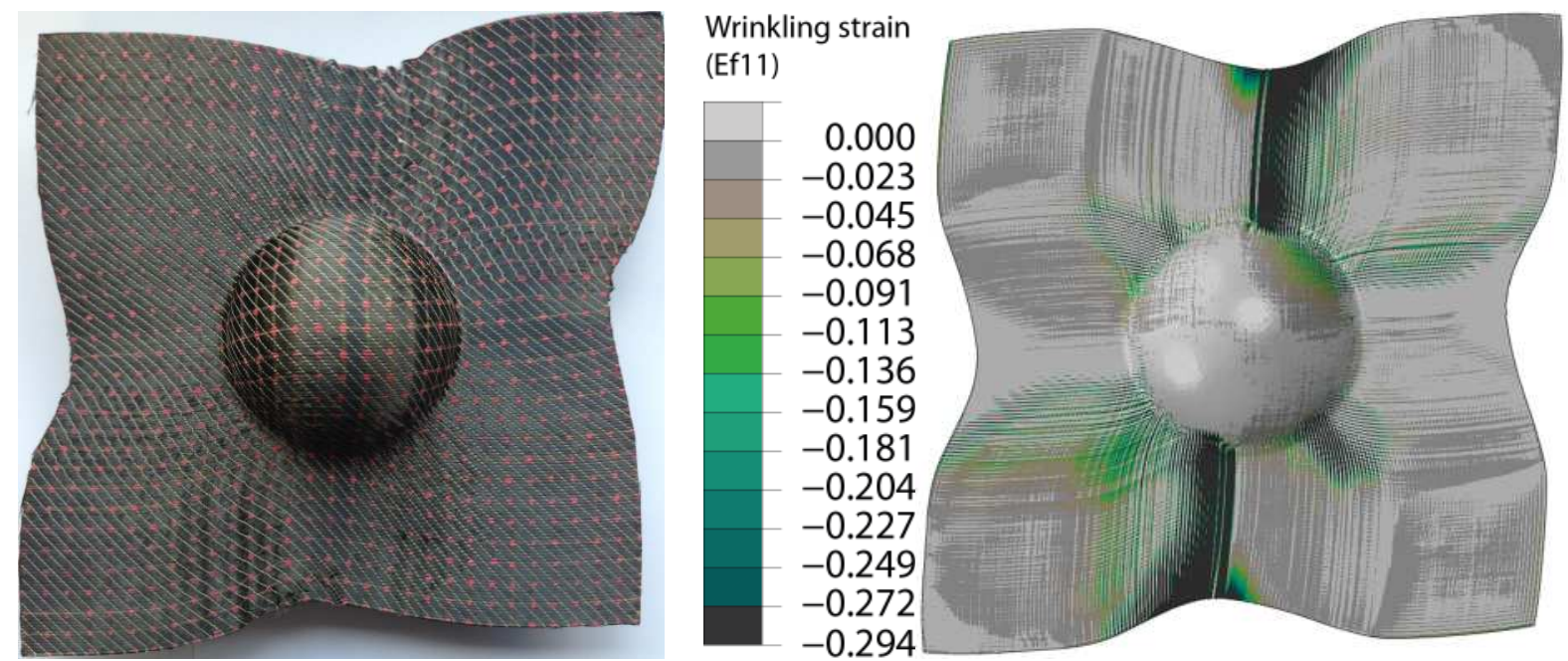

(a) Wrinkling strain of initial $0^{\circ}$ yarn (hemisphere convex side)
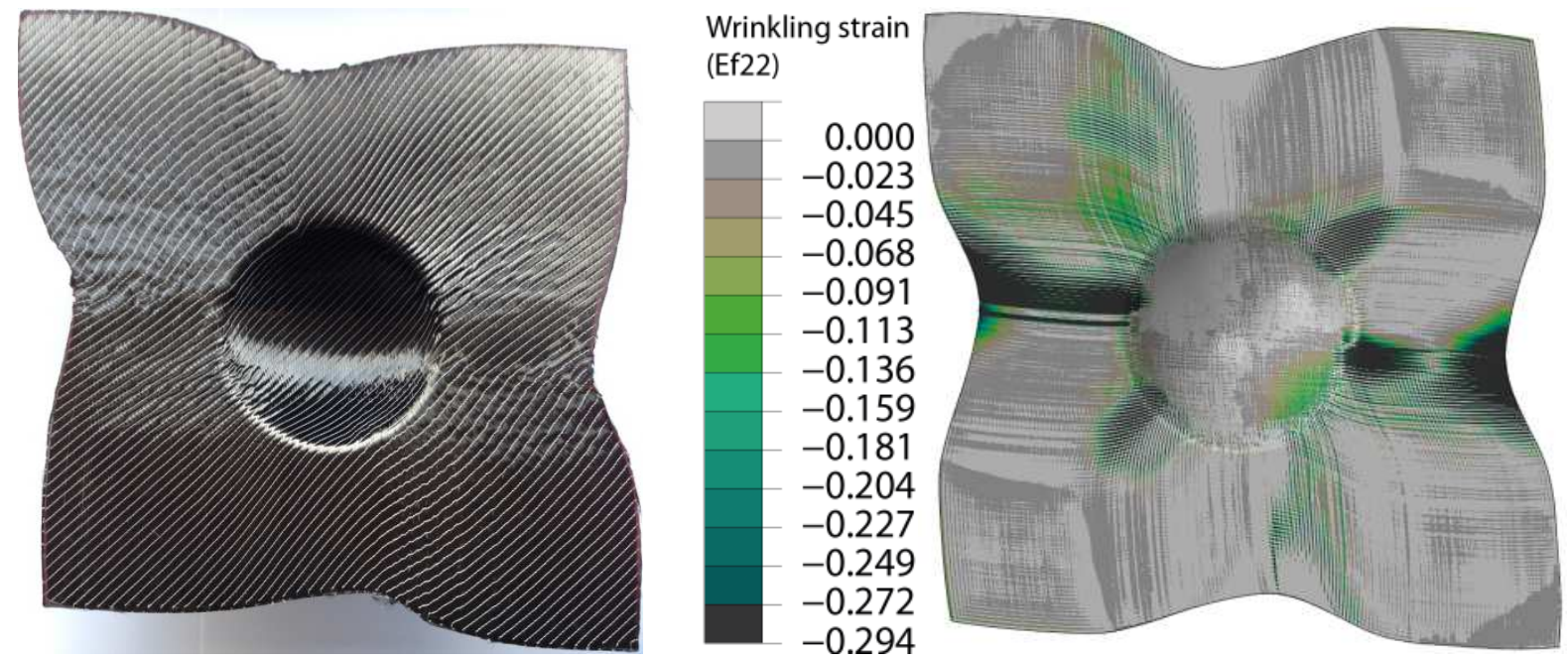

(b) Wrinkling strain of initial $90^{\circ}$ yarn (hemisphere concave side)

Figure 13: Wrinkling defect induced in NCF hemisphere forming process at a punch displacement of $50 \mathrm{~mm}$. Left: appearance of preform surfaces after forming experiment. Right: simulated in-plane strain distributions. 


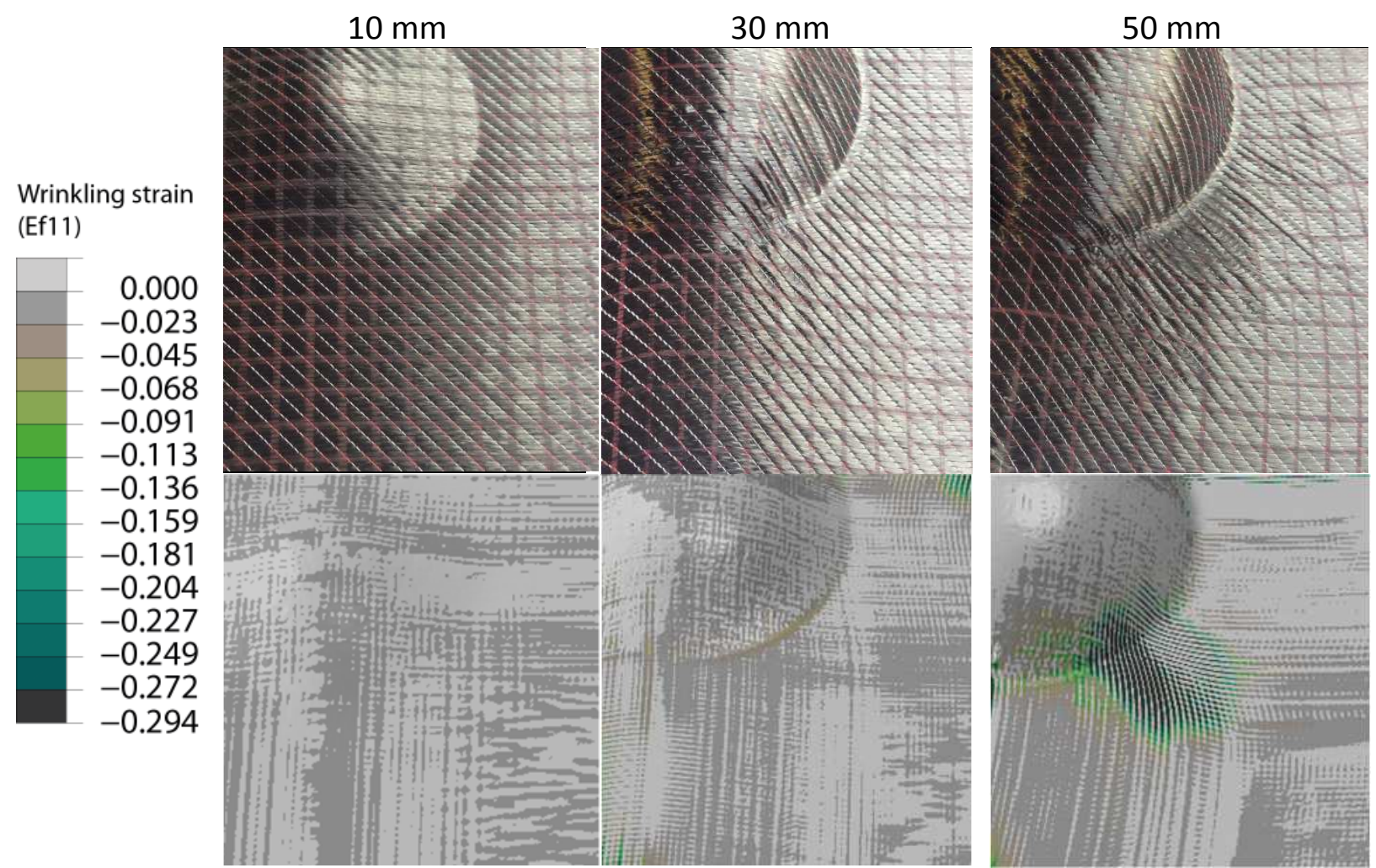

(a) Macro-scale wrinkling defects of initial $0^{\circ}$ yarn (hemisphere convex side)

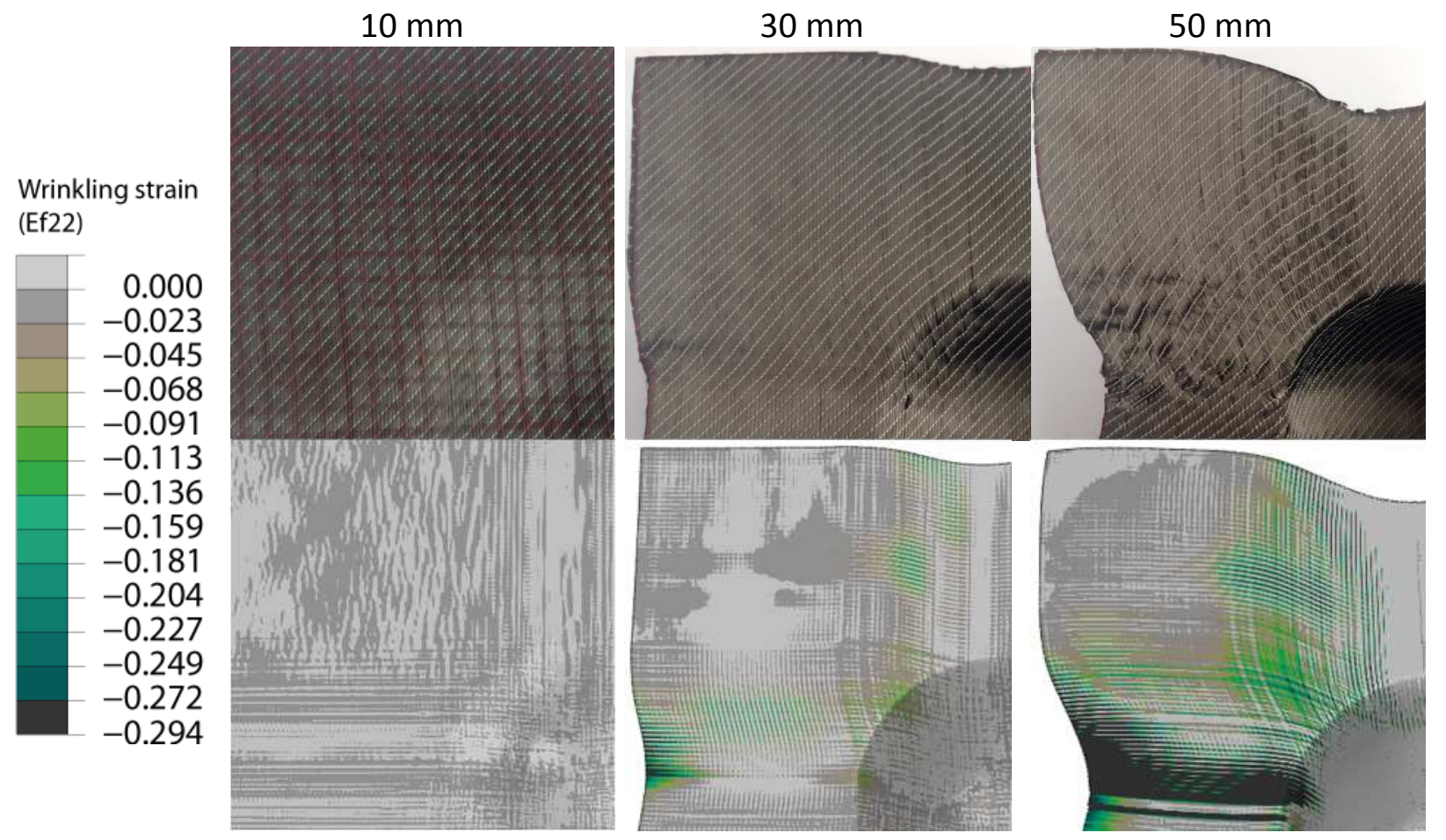

(b) Meso-scale wrinkling defects of initial $90^{\circ}$ yarn (hemisphere concave side)

Figure 14: Evolution of wrinkling defects with increasing punch displacement. Top: appearance of preform surfaces after forming experiment. Bottom: simulated in-plane strain distributions. 
Table 1: Summary of NCF material parameters (Photo shows both surfaces of NCF ply)

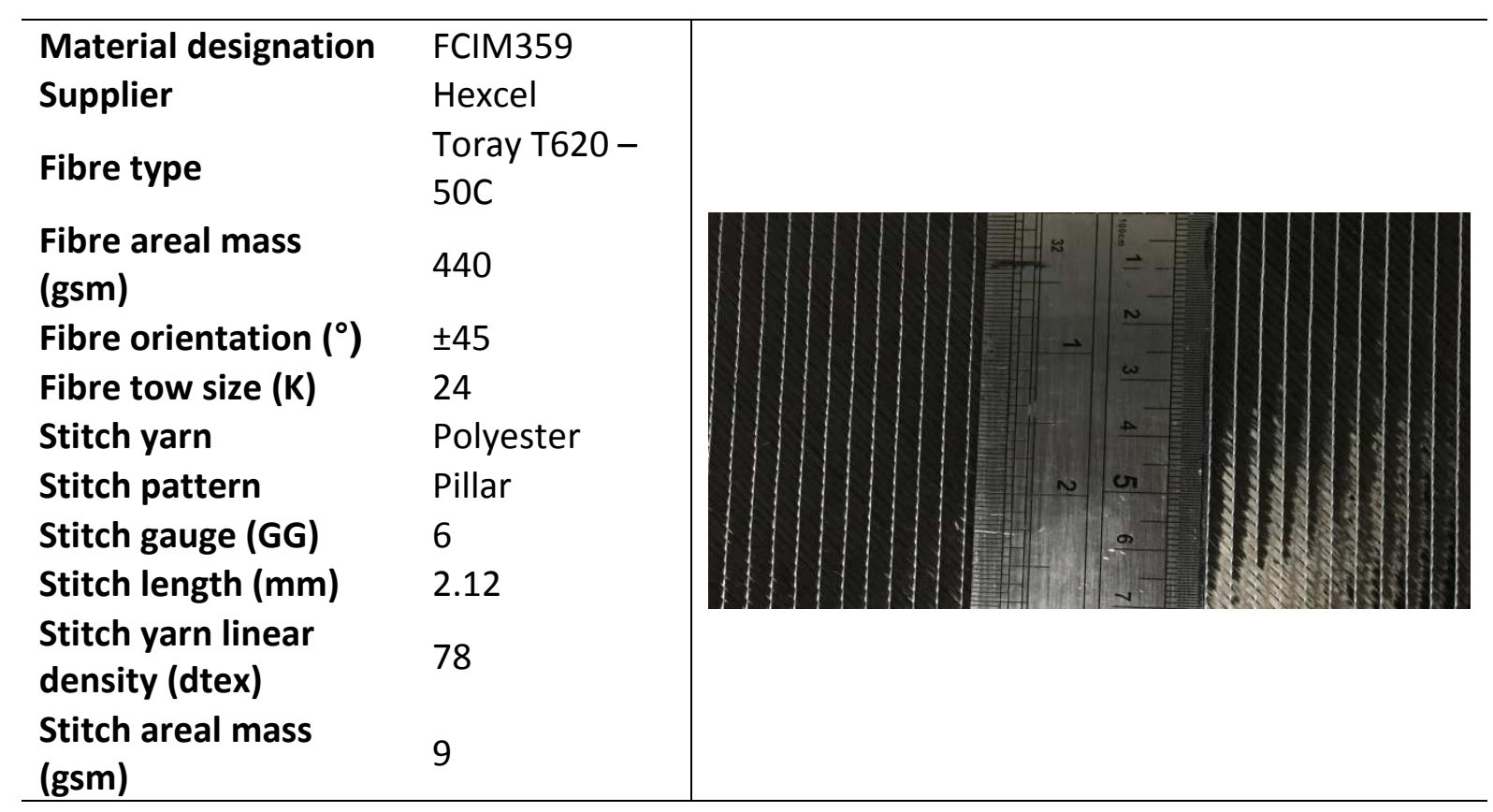

